\title{
Development of PNA microarrays for gene diagnostics with TOF-SIMS
}

\author{
H.F. Arlinghaus ${ }^{\mathrm{a},{ }^{*}, \text { M. Schröder }}{ }^{\mathrm{a}}$, J.C. Feldner ${ }^{\mathrm{a}}$, O. Brandt ${ }^{\mathrm{b}}$, \\ J.D. Hoheisel ${ }^{\mathrm{b}}$, D. Lipinsky ${ }^{\mathrm{a}}$ \\ ${ }^{a}$ Physikalisches Institut der Univerisitat Munster, Westfälische Wilhelms-Universität, \\ Wilhelm-Klemm-Str. 10, D-48149 Münster, Germany \\ ${ }^{\mathrm{b}}$ Deutsches Krebsforschungszentrum Heidelberg, Im Neuenheimer Feld 580, D-69120 Heidelberg, Germany
}

Available online 10 May 2004

\begin{abstract}
We have used time-of-flight secondary ion mass spectrometry (TOF-SIMS) to examine different immobilization processes of peptide nucleic acid (PNA) and their influence on the hybridization efficiency of unlabeled DNA fragments to complementary PNA. Two different approaches have been used to immobilize PNA onto Au surfaces. One method was to immobilize thiolated PNA in a single step reaction to the Au surface via an Au-S bond. The other method was to crosslink the $\mathrm{N}$-terminal end of the PNA to a preformed functionalized self-assembly monolayer (SAM). The influence of length and type of spacer molecules, which increase the distance between the biosensor surface and the PNA sequence, on the hybridization efficiency was also investigated. It was found that a greater spacer length leads to an increased hybridization efficiency. DNA could readily be identified by detecting $\mathrm{PO}_{2}{ }^{-}$and $\mathrm{PO}_{3}{ }^{-}$or other phosphate-containing molecules from the DNA backbone. Using TOF-SIMS a very good discrimination between complementary and non-complementary hybridized DNA sequences was achieved. It can be concluded that TOF-SIMS is a very useful technique for identifying unlabeled hybridized DNA on PNA biosensor chips. This method has the potential for providing a rapid method for DNA diagnostics and is also very suitable for studying the complexity of the immobilization and hybridization processes. Both the absence of a labeling procedure and the increase in the number of phosphate signals with increasing sequence length makes this technique especially useful for directly analyzing genomic DNA.
\end{abstract}

(C) 2004 Elsevier B.V. All rights reserved.

Keywords: DNA; PNA; TOF-SIMS; Biosensor; Microarray; Self-assembly monolayer

\section{Introduction}

In recent years, nucleic acid chip technology has been a subject of growing interest for clinical diagnostics as well as for DNA and cDNA sequencing,

\footnotetext{
* Corresponding author. Tel.: +49-251-8339064; fax: +49-251-8339063.

E-mail address: arlinghaus@uni-muenster.de (H.F. Arlinghaus).
}

partial sequencing of clones, single nucleotide polymorphism (SNP) studies, and for identification of expressed genes. Nucleic acid chips are based on sequencing by hybridization, where unknown DNA fragments are hybridized to complementary nucleic acid sequences which are immobilized in an array format on a solid surface. The main variables in this process are the attachment of the nucleic acid sequences to a solid surface (immobilization), the 
hybridization conditions, and the detection of hybridized DNA sequences.

Currently, various techniques are used to detect hybridized DNA or RNA. Most of them use PCR for amplification, and labeling procedures such as fluorescent or radioactive tags for detection. Also, a number of approaches have been made using stable isotopes as tags.

By combining peptide nucleic acid (PNA) microarray chips [1-5] as probes for hybridization of DNA sequences with time-of-flight secondary ion mass spectrometry (TOF-SIMS) for detecting complementary hybridization with high sensitivity, label-free and PCR-free DNA diagnostics should become possible. PNA is a synthesized DNA analog in which both the phosphate and the deoxyribose of the DNA backbone are replaced by a polypeptide. Nevertheless, PNA retains the ability to hybridize with complementary DNA or RNA sequences, thus allowing PNA chips to be used in the same way as DNA chips. Combined with TOF-SIMS, a technique that can identify the presence of phosphates in a molecular surface layer with high sensitivity in the attomole range, the detection of hybridization with unmodified genomic DNA becomes possible without any amplification or labeling step needed.

An additional advantage using PNA instead of DNA in microarrays is the neutral polypeptide backbone of PNA, which leads to an increased strength of the PNA/ DNA base-pairing. The lack of charge repulsion further improves the hybridization properties of DNA to PNA as compared to DNA to DNA. These improved hybridization properties are of particular importance for SNP studies.

\section{Experimental}

All experiments were carried out using a reflectronbased TOF-SIMS instrument (TOF-IV) equipped with an electron-impact primary ion gun, providing a $10 \mathrm{keV} \mathrm{Ar}^{+}$or $\mathrm{Xe}^{+}$primary ion beam to bombard the sample (primary ion dose density: $\sim 1.0 \times$ $10^{12} \mathrm{~cm}^{-2}$ ). The macroraster mode was used for the acquisition of large scale secondary ion images, as the analyzed biosensors were $20 \mathrm{~mm} \times 20 \mathrm{~mm}$ in size. This technique allows the creation of mass-resolved images of elemental and molecular distributions by stepwise movement of the sample stage and obtaining mass spectra for each pixel, with analysis being performed on sample areas which exceed the acceptance of the mass spectrometer. The image size is only limited by the possible maximum or minimum travel way of the sample stage.

\section{Results and discussion}

For the development of PNA microarrays, thiols $[3,4]$ have proven to be the molecules of choice in the formation of self-assembly monolayers (SAMs), which are the basis for PNA immobilization. We investigated different methods for the construction of these PNA microarrays on gold-coated silicon wafers. One method uses an indirect approach to immobilize PNA on a gold surface, where a thiol containing a functional end-group like a carboxylic acid or an amino group is used to build a SAM. The PNA is then attached to this SAM by using a coupling reagent like 1-ethyl-3-(3-dimethylaminopropyl)-carbodiimide hydrochloride (EDC) or disuccinimidyl carbonate (DSC), which can link an $-\mathrm{NH}_{2}$ group to a $-\mathrm{COOH}$ group or two $-\mathrm{NH}_{2}$ groups, respectively. Another more direct approach, later referred to as direct immobilization, uses PNA synthesized with a thiol linker. In this case, the PNA molecule can be directly immobilized by spotting onto a gold surface. In a second step, the remaining surface of the biosensor is covered with a layer of another thiol molecule in order to prevent DNA from associating and non-specifically binding to the gold surface.

TOF-SIMS was used to characterize the various immobilization processes which have to be iteratively optimized in order to achieve good hybridization conditions. Preliminary investigations of directly immobilized DNA and PNA fragments have shown that mass spectra of negatively charged secondary ions can be used to identify DNA and PNA fragments [2].

Following the different immobilization procedures, hybridization experiments were carried out. Mass spectra taken after such hybridization experiments are shown in Fig. 1. In these experiments, the same PNA sequence containing only cytosine and thymine (mass signal labeled as (Thy-H) ${ }^{-}$) was immobilized via the indirect approach on two different gold 

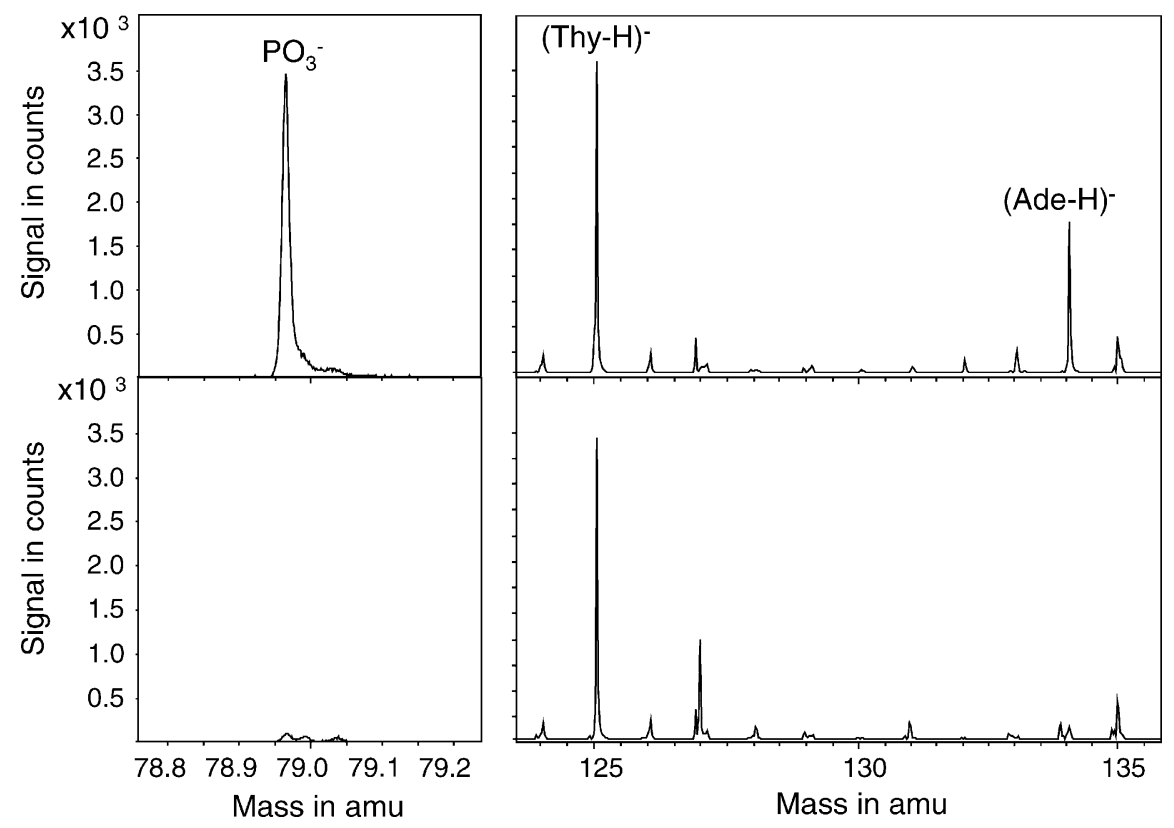

Fig. 1. Negative TOF-SIMS spectra (78.75-79.15 and 123.5-135. $5 \mathrm{amu})$ obtained from hybridization experiments where a complementary DNA sequence (3'-AAAAGGGAGAGAG-5') at the top and a non-complementary DNA sequence $\left(3^{\prime}\right.$-ACAGACACGACAC-5') at the bottom were hybridized to an immobilized PNA sequence $\left(5^{\prime}\right.$-TTTTCCCTCTCTC- $\left.3^{\prime}\right)$.

surfaces. Hybridization was carried out with two different adenine (mass signal labeled as $\left(\right.$ Ade-H) ${ }^{-}$) and guanine containing DNA sequences of which one was complementary to the PNA probe. The mass spectrum obtained from the PNA chip treated with the complementary DNA sequence (Fig. 1, top) shows that hybridized DNA can be unambiguously distinguished from the immobilized PNA by the dominant peaks of $\mathrm{PO}_{3}{ }^{-}$and $(\text {Ade- }-\mathrm{H})^{-}$. In contrast, the spectrum taken from the PNA chip treated with the noncomplementary DNA sequence (Fig. 1, bottom) only shows a prominent $(\mathrm{Thy}-\mathrm{H})^{-}$signal originating from the immobilized PNA sequence. This (Thy-H) ${ }^{-}$signal is present with similar signal intensity in both spectra. Interferences due to some minor ion peaks occurring at approximately the same mass as $\mathrm{PO}_{3}{ }^{-}$and (Ade$\mathrm{H})^{-}$are separated out by the high mass resolution of $m / \Delta m>6000$. Integration over the $\mathrm{PO}_{3}{ }^{-}$and (Ade$\mathrm{H})^{-}$peaks of this experiment results in a discrimination ratio of more than 10 between complementary and non-complementary DNAs. The best ratio observed in hybridization experiments was 200:1. The results show that the detection of unlabeled DNA oligonu- cleotides hybridized to complementary PNA is possible via the detection of negative phosphate ions $\left(\mathrm{PO}_{2}{ }^{-}\right.$and $\left.\mathrm{PO}_{3}{ }^{-}\right)$or other phosphate-containing DNA fragments in a very efficient way with TOFSIMS [4,6].

One aspect of the immobilization process influencing the hybridization efficiency comprises length and type of the spacer molecule, which is used to increase the distance between the biosensor surface and the PNA sequence. The TOF-SIMS results obtained from experiments performed on a PNA microarray are shown in Fig. 2. In this experiment, two different PNA sequences were used, one with a sequence containing only cytosine and thymine and one with a sequence containing a mixture of all four bases. From each of the two sequences, three different thiolated PNA compounds were synthesized using different spacer molecules placed between the PNA sequence and the thiol group. These different PNA molecules were immobilized directly onto a gold surface, using robotic spotting techniques [7]. The two PNA molecules with different sequences were applied in two blocks, containing 

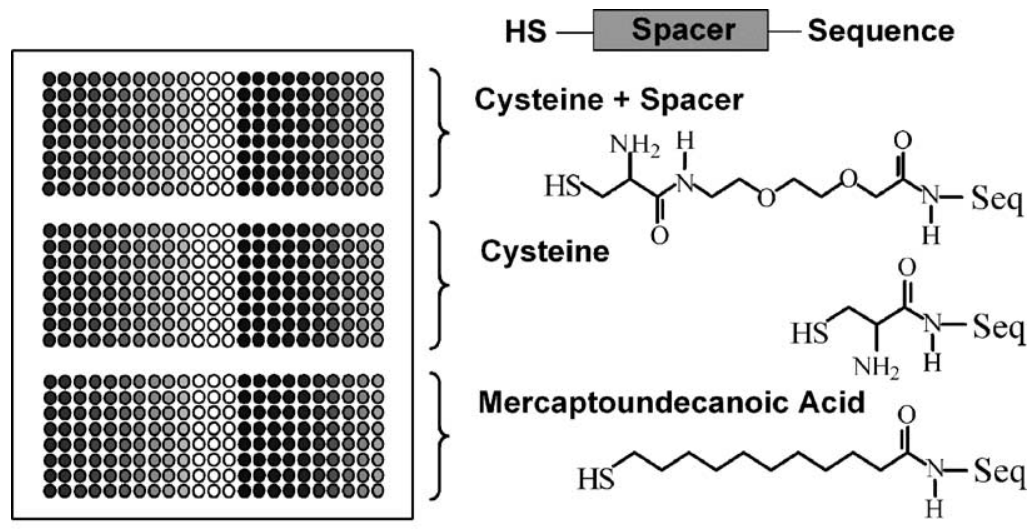

Mercaptoundecanoic Acid
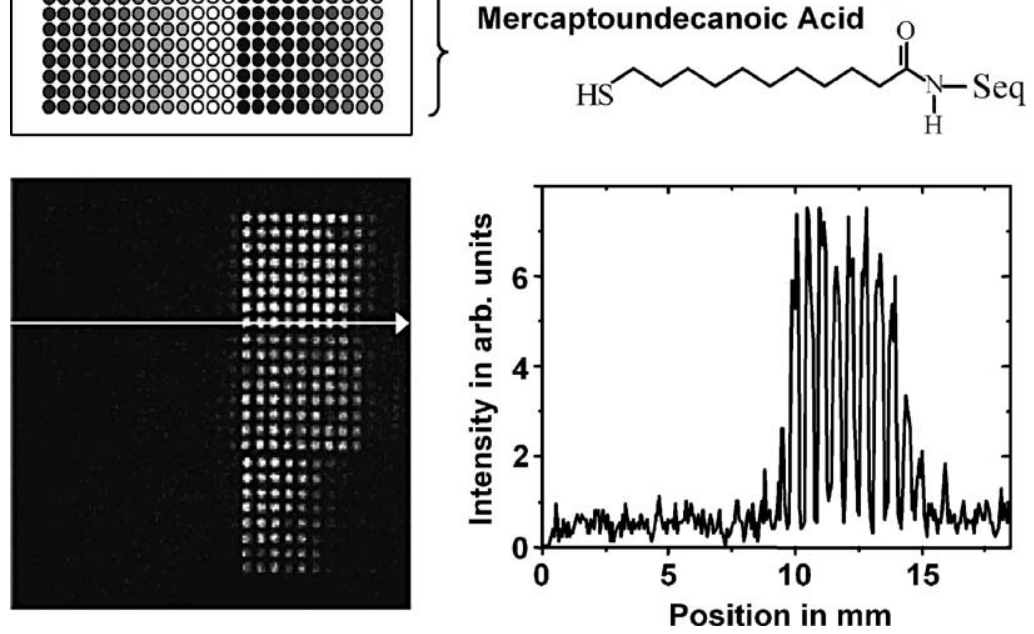

Fig. 2. TOF-SIMS image (159 amu) and a line scan obtained from a PNA microarray chip hybridized with unlabeled DNA using three different types of spacer. The position where the line scan was taken is marked by an arrow. PNA sequences on the left: 5'-AGCTTACGGATCA-3' and on the right: 5'-TTCTCCCTCTCTC-3'; hybridized DNA sequence: 5'-TTCGATAGCAGCTAAATTCTGAGAGAGGGAGAATTCTACAATAACTGCGA-3'. PNA concentration changes from darker (highest concentration) to lighter (lowest concentration) colored dots: $160,140,120,100,80,60,40,20,10,5 \mu \mathrm{M}$; substrate size: $20 \mathrm{~mm} \times 20 \mathrm{~mm}$; spot size diameter: $360 \mu \mathrm{m}$.

three different spacer molecules in rows, with eight duplications each. In each row the PNA was applied in a concentration gradient, ranging from 160 to $5 \mu \mathrm{M}$ (from left to right).

An unlabeled DNA oligonucleotide was hybridized to the complementary PNA sequence immobilized on the right-hand side (Fig. 2). TOF-SIMS analysis shows that hybridized unlabeled DNA could be detected with good discrimination by a phosphorcontaining fragment of the DNA backbone $(159 \mathrm{amu})$ at the complementary PNA positions. The highest signal was obtained at positions where PNA with the longest spacer length was immobilized. The line scan also shows that at these positions there are no significant changes in signal intensity between the higher concentrations of the PNA probe.
Note that only a small fraction of a monolayer is needed for analysis using an $\mathrm{Ar}^{+}$ion beam with a focus diameter of $30 \mu \mathrm{m}$. Additional experiments showed that it is possible to detect DNA in an area of less than $100 \mathrm{~nm}$ in diameter using a focussed $\mathrm{Ga}^{+}$ion beam, which corresponds to attomole sensitivity.

\section{Conclusion}

The data clearly show that TOF-SIMS is a powerful technique to unambiguously identify unlabeled DNA hybridized to PNA microarray chips by detecting phosphate or phosphate-containing molecules resulting from DNA fragmentation. TOF-SIMS is also 
suitable for studying the immobilization and hybridization process. Utilizing unlabeled DNA has several advantages over using fluorescent and radioactive labeling procedures. These advantages are a higher signal-to-noise ratio, higher sensitivity, absence of a labeling or amplification procedure, and the possibility of direct analysis of hybridized genomic DNA. Particularly, the increase in the number of phosphates with increasing sequence length will be advantageous for sequence analysis of genomic DNA.

\section{Acknowledgements}

This work was supported by the BMBF under Contract No. 13N7858.

\section{References}

[1] H.F. Arlinghaus, M.N. Kwoka, K.B. Jacobson, Anal. Chem. 69 (1997) 3747.

[2] H.F. Arlinghaus, C. Hoppener, J. Drexler, in: A. Beminghoven, P. Bertrand, H.-N. Migeon, H.W. Werner (Eds.), Secondary Ion Mass Spectrometry SIMS XII, Elsevier, Amsterdam, 2000, p. 951.

[3] H.F. Arlinghaus, M. Ostrop, O. Friedrichs, J. Feldner, U. Gunst, D. Lipinsky, Surf. Interf. Anal. 33 (2002) 35.

[4] J.C. Feldner, M. Ostrop, O. Friedrichs, S. Sohn, D. Lipinsky, U. Gunst, H.F. Arlinghaus, Appl. Surf. Sci. 203 (2003) 722.

[5] S. Matysiak, F. Reuthner, J.D. Hoheisel, BioTechniques 31 (2001) 896

[6] H.F. Arlinghaus, M. Ostrop, O. Friedrichs, J. Feldner, Appl. Surf. Sci. 203 (2003) 689.

[7] J. Weiler, H. Gausepohl, N. Hauser, O.N. Jensen, J.D. Hoheisel, Nucl. Acids Res. 25 (1997) 2792. 